\title{
Theoretical design of highly correlated electron states in delafossite heterostructures
}

\author{
Frank Lechermann ${ }^{1}{ }^{1}$ and Raphael Richter ${ }^{2}$ \\ ${ }^{1}$ I. Institut für Theoretische Physik, Universität Hamburg, D-20355 Hamburg, Germany \\ ${ }^{2}$ Institut für Technische Thermodynamik, Deutsches Zentrum für Luft- und Raumfahrt, D-70569 Stuttgart, Germany
}

(Received 19 December 2019; revised manuscript received 28 February 2020; accepted 3 March 2020; published 20 March 2020)

\begin{abstract}
Delafossites represent natural heterostructures which can host rather different electronic characteristics in their constituting layers. The design of heterostructure architectures highlighting the competition between such varying layer properties is promising from the viewpoint of basic research as well as for future technological applications. By means of the combination of density functional theory and dynamical mean-field theory, we here unveil the formation of highly correlated electron states in delafossite heterostructures built from metallic $\mathrm{PdCrO}_{2}$ and insulating $\mathrm{AgCrO}_{2}$. Due to the sophisticated coupling between layers of strong and of weak internal electron-electron interaction, correlation-induced semimetals at ambient temperature and doped Mott insulators at lower temperature are predicted. The unique electronic structure of delafossite heterostructures opens a door to research on challenging quantum matter.
\end{abstract}

DOI: 10.1103/PhysRevResearch.2.013352

\section{INTRODUCTION}

Delafossite oxides [1] $A B \mathrm{O}_{2}$, where $A$ and $B$ are different metallic elements, have gained ample renewed interest, mainly from two directions. First, the dominant part of these compounds is insulating, and technologically promising due to their potential as transparent conducting oxides [2] as well as their possibly multifunctional character, e.g., by showing magnetic/ferroelectric response [3,4] and high potential for photocatalytic applications [5]. Second, the small subgroup of metallic delafossites usually displays an enormously high electric conductivity, belonging to the most-conductive metals at room temperature [6,7]. General key signatures of delafossite crystal lattices are the triangular-coordinated planes and especially the dumbbell $\mathrm{O}-A-\mathrm{O}$ bonds along the $c$ axis. The layered $R \overline{3} m$ architecture of alternating $A^{+}$and $B^{3+} \mathrm{O}_{2}$ planes gives rise to a "natural heterostructure," i.e., the different planes may host a rather different electronic character. Tuning and designing the different aspects of delafossites by further technological heterostructuring thus may open a route to challenging new materials with exceptional properties.

Among these fascinating materials, the $\mathrm{PdCrO}_{2}$ compound stands further out as it unites high metallicity from the $\mathrm{Pd}^{+}\left(4 d^{9}\right)$ planes with Mott-insulating $\mathrm{CrO}_{2}$ layers [8-11]. Replacing Pd by neighboring Ag formally leads to a closed $4 d^{10}$ shell and, nonsurprisingly, $\mathrm{AgCrO}_{2}$ is indeed insulating with a technologically interesting band gap of $1.68 \mathrm{eV}$ [5]. In both delafossites, the localized $\mathrm{Cr}$ spins order in an antiferromagnetic $120^{\circ}$ manner below Néel temperatures $37.5 \mathrm{~K}$

Published by the American Physical Society under the terms of the Creative Commons Attribution 4.0 International license. Further distribution of this work must maintain attribution to the author(s) and the published article's title, journal citation, and DOI.
$\left(\mathrm{PdCrO}_{2}\right)$ and $21 \mathrm{~K}\left(\mathrm{AgCrO}_{2}\right)$. Moreover, the palladium delafossite shows an unconventional anomalous Hall effect [12] at low temperature $T$, whereas the silver delafossite displays multiferroic properties [3]. While both individual compounds are of prominent interest of their own, we want to open another path in delafossite research in this work. By using the oxide-heterostructure concept, the goal is to theoretically further engineer the unique layer-triggered competition between itinerant and insulating tendencies in these $\mathrm{CrO}_{2}$-based delafossites. As we will show, this manipulation enables the stabilization of even more intriguing electronic phases and drives the challenge of the common metal-versus-insulator paradigm to another level. We study delafossite heterostructures $\mathrm{Pd}_{n} \mathrm{Ag}_{m} \mathrm{CrO}_{2}$ with $n(m)=1,2$ planes of $\mathrm{Pd}(\mathrm{Ag})$ in the primitive cell, and as in the conventional bulk compounds, separated by $\mathrm{CrO}_{2}$ layers. Thus three different artificial heterostructures are realized mediating between the natural heterostructures $\mathrm{PdCrO}_{2}$ and $\mathrm{AgCrO}_{2}$, namely $\mathrm{Pd}_{2} \mathrm{AgCrO}_{2}$ (P2A), $\mathrm{PdAgCrO}_{2}$ (PA), and $\mathrm{PdAg}_{2} \mathrm{CrO}_{2}$ (PA2) (see Fig. 1). In order to cope with the demanding problem involving the competition between itinerant and band-insulating as well as Mott-insulating layers, the elaborate charge self-consistent combination [13-15] of density functional theory (DFT) and dynamical mean-field theory (DMFT) is put into practice to elucidate the correlated electronic structure, respectively. At room temperature, the three designed heterostructures are predicted to be correlation-induced semimetals with singular electronic characteristics. Lowering the temperature leads to a doped-Mott insulator scenario at low energy.

\section{THEORETICAL APPROACH}

A charge self-consistent DFT + DMFT approach [15] is employed. The mixed-basis pseudopotential method [16,17], based on norm-conserving pseudopotentials with a combined basis of localized functions and plane waves, is used for the 

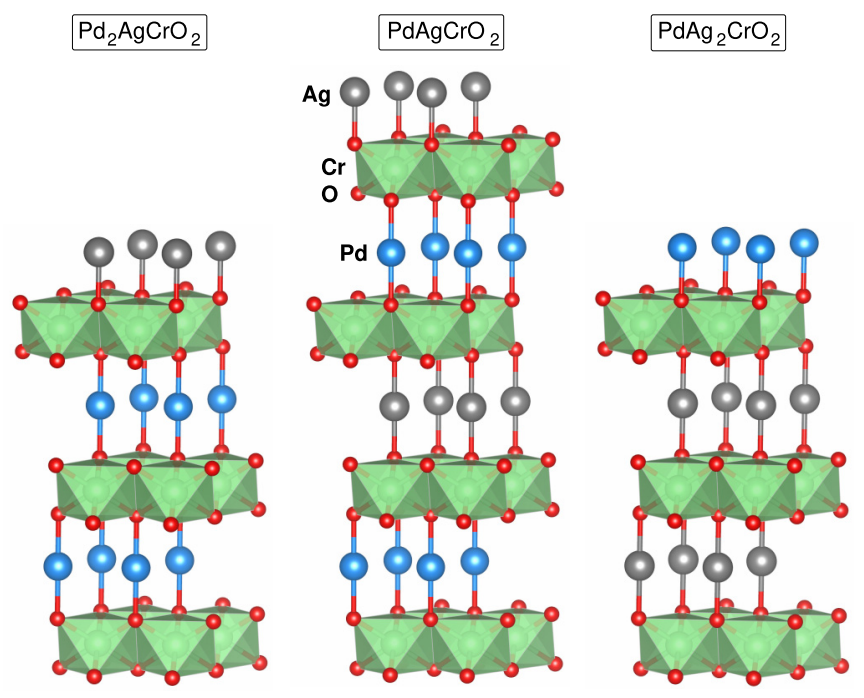

FIG. 1. Crystal structures of the designed delafossite heterostructures with stacking along the $c$ axis. $\mathrm{Pd}_{2} \mathrm{AgCrO}_{2}, \mathrm{PdAgCrO}_{2}$, and $\mathrm{PdAg}_{2} \mathrm{CrO}_{2}$ (from left to right). Pd: blue; $\mathrm{Ag}$ : gray; $\mathrm{Cr}$ : green; $\mathrm{O}$ : red.

DFT part with the generalized-gradient approximation in the form of the PBE functional [18]. Within the mixed basis, localized functions for $\operatorname{Cr}(3 d), \operatorname{Pd}(4 d)$, and $\operatorname{Ag}(4 d)$ states as well as for $\mathrm{O}(2 s)$ and $\mathrm{O}(2 p)$ are utilized to reduce the plane-wave energy cutoff. The latter is chosen $E_{\text {cut }}=20 \mathrm{Ry}$ for the bulk structures and $E_{\text {cut }}=16 \mathrm{Ry}$ for the heterostructures. A k-point mesh of $13 \times 13 \times 13$ partition is utilized for the bulk structures and of $11 \times 11 \times 3$ partition for the heterostructures.

The correlated subspace for the DMFT part consists of the effective $\mathrm{Cr}(3 d)$ Wannier-like functions as obtained from the projected-local-orbital formalism [19,20], using as projection functions the linear combinations of atomic $3 d$ orbitals, diagonalizing the $\mathrm{Cr}(3 d)$ orbital-density matrix. A fiveorbital Slater-Kanamori Hubbard Hamiltonian governs the interacting electrons, parametrized by a Hubbard $U$ and a Hund's exchange $J_{\mathrm{H}}$. The coupled single-site DMFT impurity problems in the given structures are solved by the continuoustime quantum Monte Carlo scheme [21,22] as implemented in the TRIQS package [23,24]. A double-counting correction of fully localized-limit type [25] is applied. No Hubbard interactions are applied to $\operatorname{Pd}(4 d)$ and $\operatorname{Ag}(4 d)$. Such interactions are already expected much smaller than on $\operatorname{Cr}(3 d)$ and then furthermore their effect onto nominal $d^{9}\left(d^{10}\right)$ of $\mathrm{Pd}(\mathrm{Ag})$ should be weak in the metallic-layer architecture. Thus restricting the explicit DMFT-impurity treatment to the $\mathrm{Cr}$ sites is sound to study key correlation effects.

To obtain the spectral information, analytical continuation from Matsubara space via the maximum-entropy method as well as the Padé method is performed. In the former case, the k-integrated Bloch Green's functions are continued, whereas in the latter case the local $\mathrm{Cr}$ self-energies are continued. If not otherwise stated, the maximum-entropy scheme is chosen to reveal k-integrated spectral functions and the Padé scheme is chosen to obtain k-resolved spectral functions. Note moreover that the k-resolved spectral functions are computed on a dense mesh along high-symmetry lines and in the $k_{z}=0$ plane of reciprocal space. For this purpose, the converged real-frequency local self-energies from the Padé scheme, and the self-consistent projected-local-orbitals recomputed on the dense mesh, are utilized to obtain well-resolved Green's functions in $\mathbf{k}$ space.

The DFT+DMFT calculations are conducted by setting the system temperature to room temperature $T=290 \mathrm{~K}$, if not otherwise stated. Paramagnetism is assumed in all the computations. Furthermore, in the following, if not otherwise stated, all shown spectral data is obtained from charge selfconsistent DFT+DMFT calculations.

\section{RESULTS}

\section{A. $\mathrm{PdCrO}_{2}$ and $\mathrm{AgCrO}_{2}$}

To set the stage, we first briefly focus on the electronic structure of the natural bulk systems $\mathrm{PdCrO}_{2}$ and $\mathrm{AgCrO}_{2}$. Experimental lattice parameters [1,5] $a=2.930 \AA$ and $c=18.087 \AA$ for $\mathrm{PdCrO}_{2}$ as well as $a=2.985 \AA$ and $c=18.510 \AA$ for $\mathrm{AgCrO}_{2}$ are used. The internal degree of freedom $z$, governing the oxygen distance to the $A^{+}$ plane, is obtained from DFT structural optimization, reading $z=0.1101(0.1095)$ for $\mathrm{PdCrO}_{2}\left(\mathrm{AgCrO}_{2}\right)$. Figures 2 and 3 present a summary of the spectral characteristics. For $\mathrm{PdCrO}_{2}$, local Coulomb interactions on the chromium site of $U=3 \mathrm{eV}$ and $J_{\mathrm{H}}=0.7 \mathrm{eV}$ prove adequate as discussed in Ref. [10].

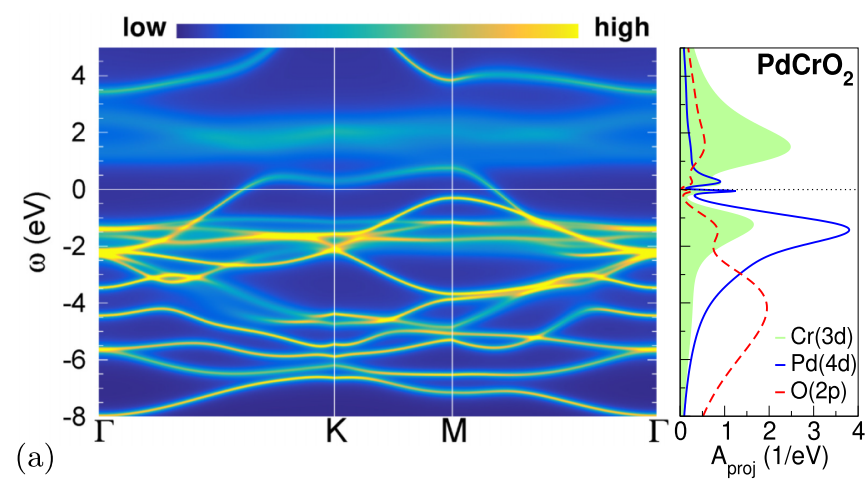

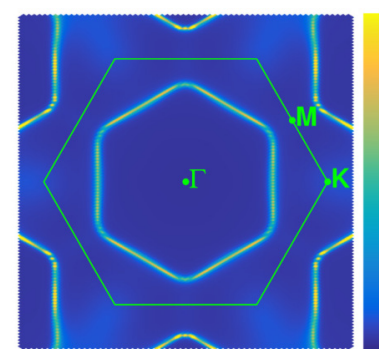

(b)

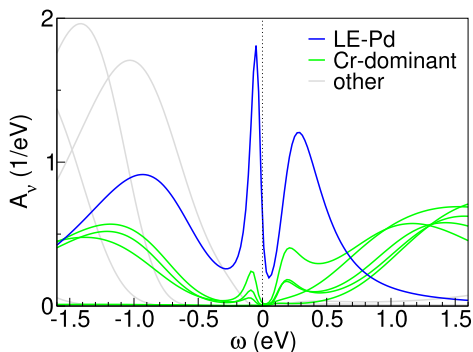

(c)
FIG. 2. Spectral data of $\mathrm{PdCrO}_{2}$. (a) Spectral function $A(\mathbf{k}, \omega)$ along high-symmetry lines in the $k_{z}=0$ plane of reciprocal space (left) and k-integrated site- and orbital-projected spectral function (right). (b) Fermi surface for $k_{z}=0$ within the first Brillouin zone (green hexagon) and (c) k-integrated Bloch contribution $A_{v}(\omega)$ (i.e., each individual curve marks a different Bloch index $v$ ) with characterization of site dominance. Note that the following figures use the same normalized color scale for the k-resolved spectral function as (a) and (b). 

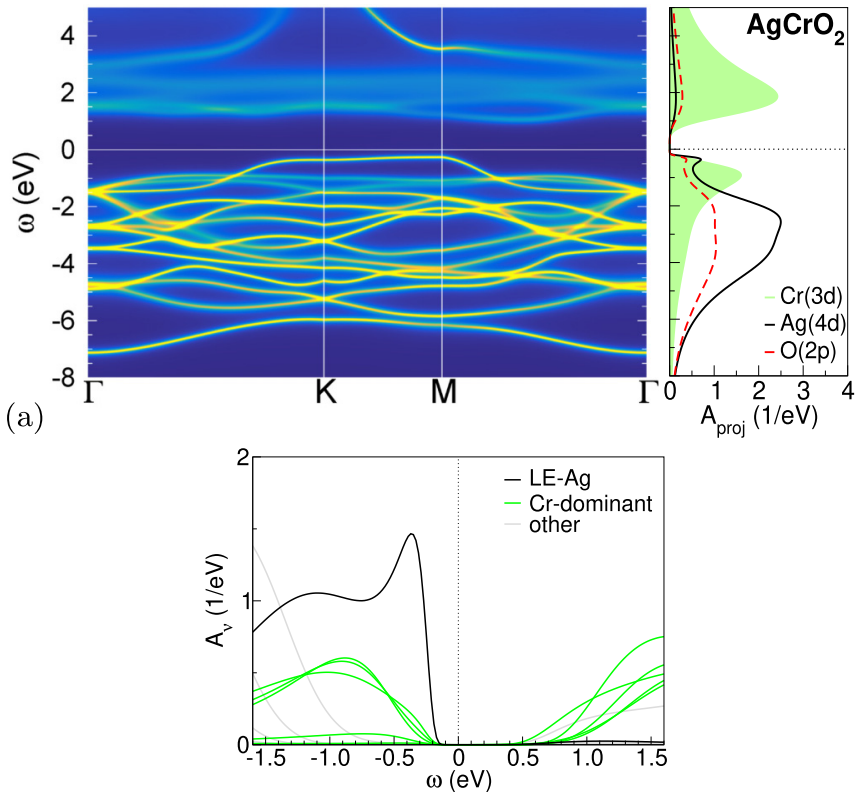

(b)

FIG. 3. Spectral data of $\mathrm{AgCrO}_{2}$. (a) Spectral function $A(\mathbf{k}, \omega)$ as in Fig. 2(a). (b) k-integrated Bloch contribution $A_{v}(\omega)$ as in Fig. 2(c).

The system is metallic with a single conducting Pd-dominated (cPd) quasiparticle dispersion at the Fermi level $\varepsilon_{\mathrm{F}}$ and Mottinsulating $\mathrm{CrO}_{2}$ layers [see Fig. 2(a)]. The cPd dispersion is dominantly formed by a linear combination of in-plane $d_{x^{2}-y^{2}}$ and $d_{x y}$, as well as by some $d_{z^{2}}$ contribution (see Ref. [10] for more details). Note that the $\mathbf{k}$-integrated projected spectral function $A_{\text {proj }}(\omega)$ still displays sizable Cr weight at low energy, associated with the cPd band. Thus intricate hybridization between the conducting and the Mott-insulating layers is at play [10]. The single-sheet Fermi surface, shown in Fig. 2(b), is of hole kind and of hexagonal shape, in line with photoemission $[9,26]$. It is furthermore instructive to visualize the spectral functions $A_{v}(\omega)$ with Bloch index $v$. The function $A_{v}(\omega)$ amounts to $\mathbf{k}$-integrated spectral weight of the correlated system per Bloch state, i.e., it provides the spectral information of the correlated material as represented in Bloch Hilbert space. The total spectral function then reads $A_{\text {tot }}(\omega)=$ $\sum_{v} A_{v}(\omega)$. Figure 3(c) shows that, dominantly, there is a single- $v$ low-energy Pd-dominated (LE-Pd) contribution at the Fermi level. Be still aware that the complete cPd spectral weight consists of the low-energy $v$ sum of all Bloch spectra.

In the case of $\mathrm{AgCrO}_{2}$, the quasi-closed-shell character of $\mathrm{Ag}$ reduces the screening within the $\mathrm{CrO}_{2}$ subsystem and we therefore increased the Hubbard interaction on $\mathrm{Cr}$ to $U=4 \mathrm{eV}$. Figures 3(a) and 3(b) exhibit that the $\mathrm{CrO}_{2}$ layers remain Mott insulating, but the previously conducting band becomes completely filled and thus the system as a whole insulating. Hence $\mathrm{AgCrO}_{2}$ is a combined band-Mott insulator. The obtained charge gap of $\sim 1.8 \mathrm{eV}$ is in good agreement with experiment. Note that the valence-band maximum is of mixed Ag, $\mathrm{O}$ character, while the conduction-band minimum is of dominant $\mathrm{Cr}$ upper-Hubbard band character. Notably, whereas the $\mathrm{Pd}(4 d)$ states in $\mathrm{PdCrO}_{2}$ align with the $\mathrm{Cr}$ lower Hubbard band, the $\operatorname{Ag}(4 d)$ states align with $\mathrm{O}(2 p)$.

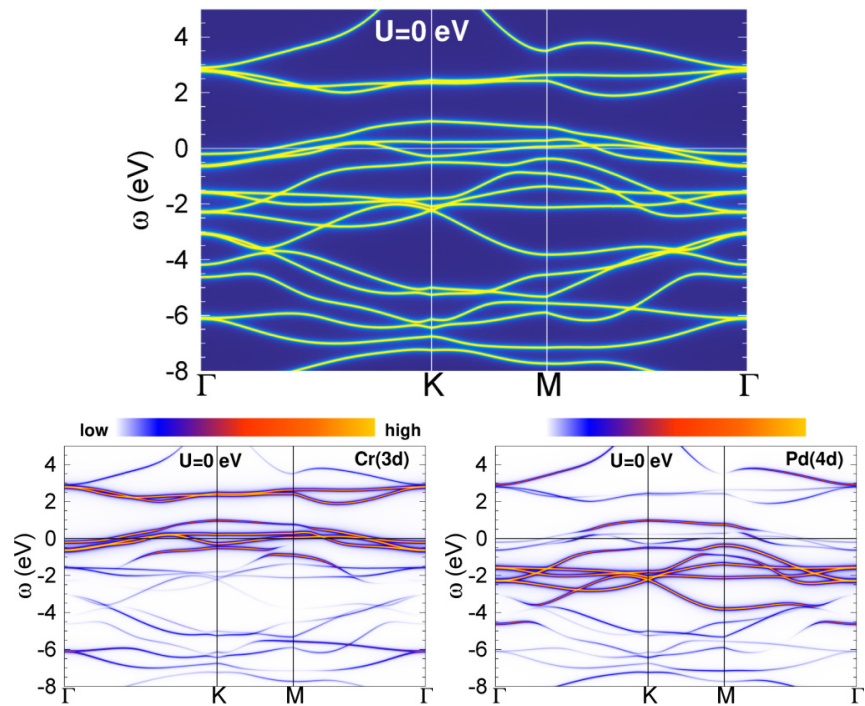

(a)

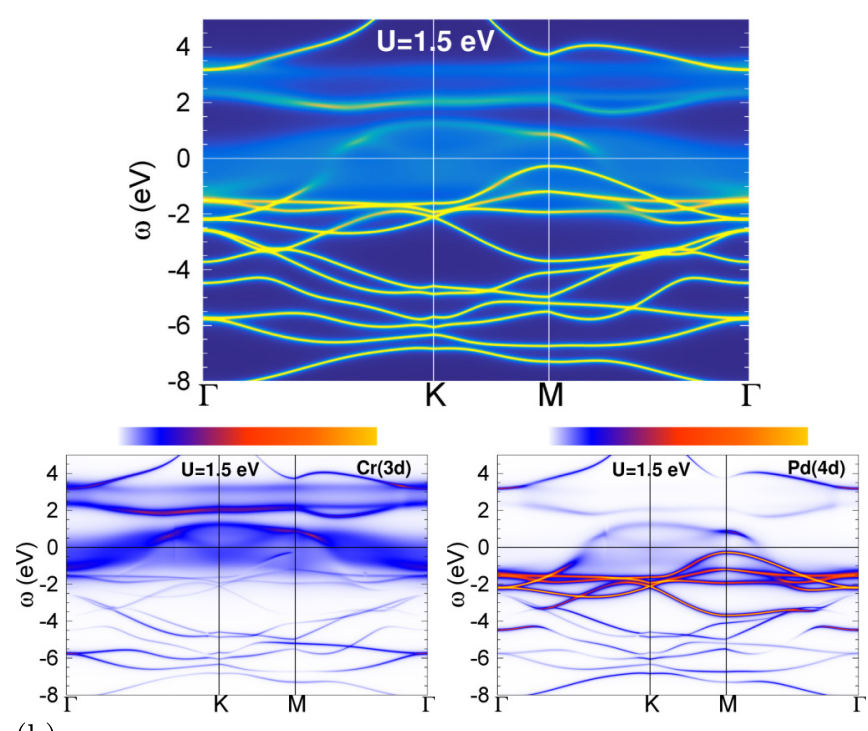

(b)

FIG. 4. k-resolved spectral function (top) as well as $\mathrm{Cr}(3 d)$ - and $\mathrm{Pd}(4 d)$-resolved spectral-function weight (bottom) of $\mathrm{PdCrO}_{2}$ for (a) $U=J_{\mathrm{H}}=0$ (i.e., DFT band structure) and for (b) $U=1.5 \mathrm{eV}$ and $J_{\mathrm{H}}=0.35 \mathrm{eV}$.

Let us comment on another important aspect, namely the evolution from the DFT electronic structure towards the fully interacting one. When turning on interactions, the electronic spectrum in $\mathrm{CrO}_{2}$-based delafossites does not evolve from a noninteracting(-like) band structure to a Mott-insulating spectrum, as observed for common Mott insulators [27]. For instance, in $\mathrm{PdCrO}_{2}$, the metallic band structure evolves to another metallic spectrum, both spectra with weakly correlated looking dispersions at low energy. This interaction evolution takes place in a highly nontrivial way, as depicted in Fig. 4. In the DFT $\left(U=J_{\mathrm{H}}=0\right)$ case, the three Cr-based $t_{2 g}$ bands cross the Fermi level and the later cPd band is still completely filled well below $\varepsilon_{\mathrm{F}}$, as visualized from the orbital-resolved spectral-function weights at the bottom of Fig. 4(a). For $U=$ $1.5 \mathrm{eV}$ and $J_{\mathrm{H}}=0.35 \mathrm{eV}$ (i.e., half the interaction strength of 
the true system), the $\mathrm{CrO}_{2}$ layers are not yet Mott insulating and the former Cr-based $t_{2 g}$ bands are now strongly incoherent even close to the Fermi level. Furthermore, the later cPd band also becomes very incoherent when entering the low-energy regime [cf. Fig. 4(b)]. These incoherent features may be observed from the spreading of the spectral weight (i.e., "bluriness") leading to a (near) disappearance of the original well-defined dispersions. Thus, intriguingly, $\mathrm{PdCrO}_{2}$ at hypothetical smaller $U$ is a very incoherent metal (or "bad metal"), apparently evading Fermi-liquid characteristics and seemingly in conflict with Luttinger's theorem. Coherence in the cPd band is reached at larger $U$ when the $\mathrm{CrO}_{2}$ layers are Mott insulating, which again underlines the intricate coupling between the $\mathrm{Pd}$ and the $\mathrm{CrO}_{2}$ layers. Substitutional doping of the $\mathrm{Cr}$ site by isovalent Mo could be a way to reach a lower effective $U$ value in the system.

\section{B. Designed heterostructures}

For the heterostructures $\mathrm{Pd}_{n} \mathrm{Ag}_{m} \mathrm{CrO}_{2}$, supercells of $1 \times 1 \times 3$ kind (12 basis atoms) for $n \neq m$ and of $1 \times 1 \times 2$ kind (six basis atoms) for $n=m=1$ are constructed (see Fig. 1). The respective supercell lattice parameters are obtained from linear interpolation of the bulk parameters and the atomic positions are structurally optimized within DFT. The Hubbard $U$ on the $\mathrm{Cr}$ site is also chosen to result from a linear interpolation of the respective bulk values, i.e., $J_{\mathrm{H}}=0.7 \mathrm{eV}$ and $U(\mathrm{P} 2 \mathrm{~A})=3.33 \mathrm{eV}, U(\mathrm{PA})=3.50 \mathrm{eV}$, and $U(\mathrm{PA} 2)=$ $3.67 \mathrm{eV}$.

\section{At room temperature: $T=290 \mathrm{~K}$}

In order to first address the phase stability, the electronic formation energy

$$
\begin{aligned}
E_{\text {form }}^{(n m)}= & E_{\text {tot }}\left(\mathrm{Pd}_{n} \mathrm{Ag}_{m} \mathrm{CrO}_{2}\right) \\
& -n E_{\text {tot }}\left(\mathrm{PdCrO}_{2}\right)-m E_{\mathrm{tot}}\left(\mathrm{AgCrO}_{2}\right),
\end{aligned}
$$

where $E_{\text {tot }}$ are the total energies either in DFT or DFT + DMFT, may be considered. Note that here, we aligned the plane-wave energy cutoff $E_{\text {cut }}$ to obtain comparable total energies. Within DFT, all designed heterostructures yield a

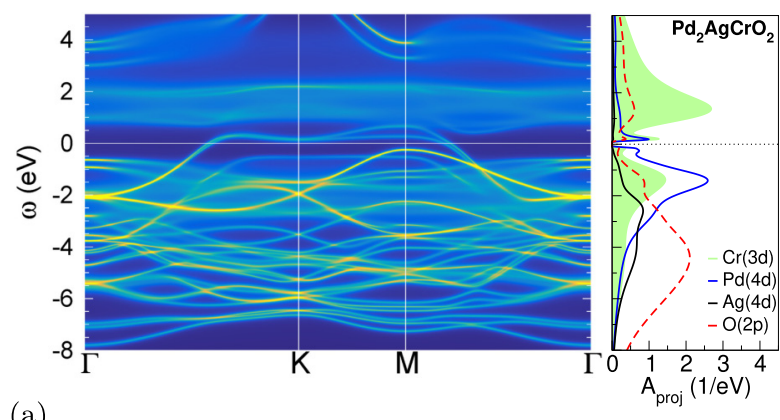

(a)

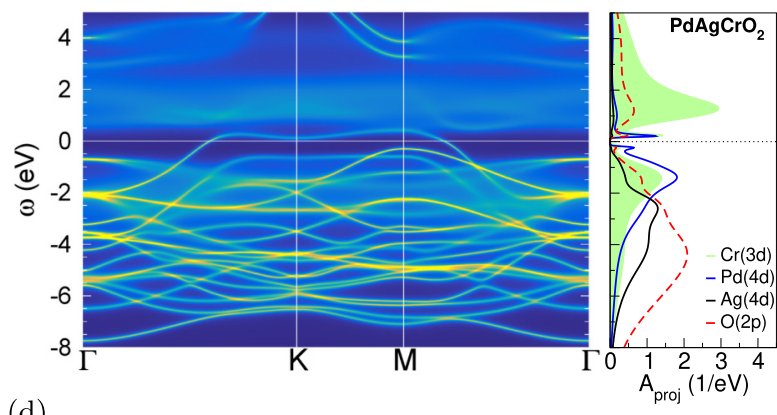

(d)

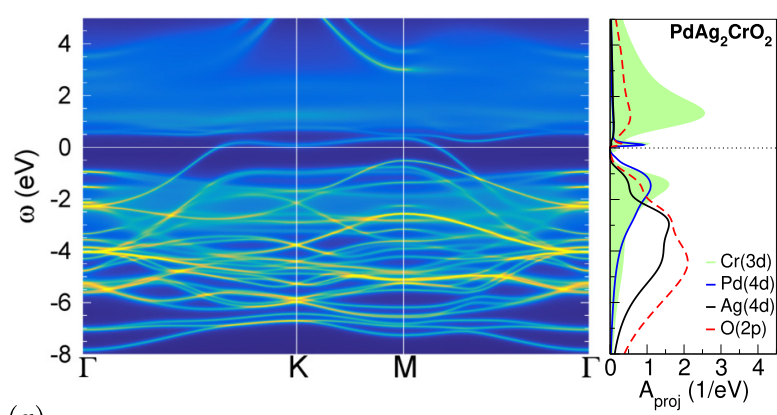

(g)

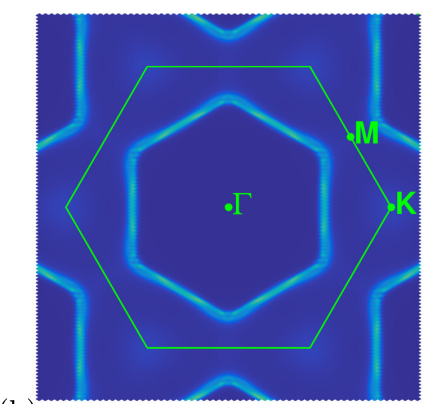

(b)

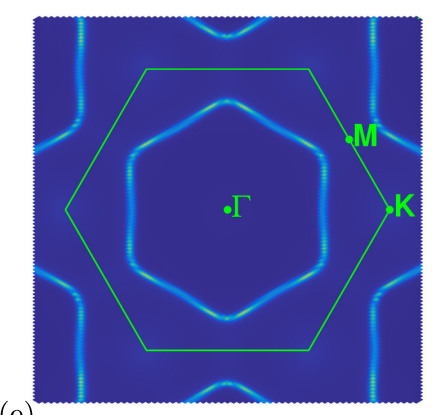

(e)

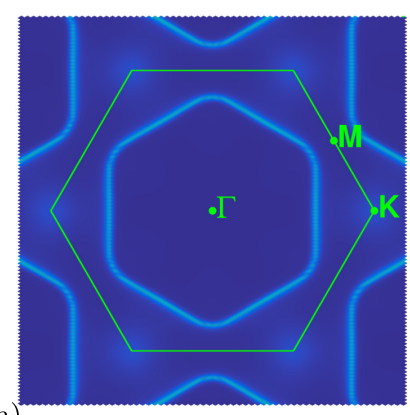

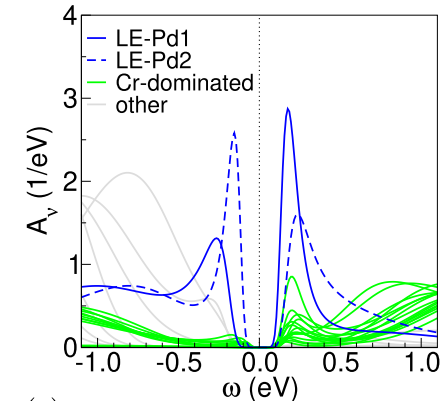

(c)

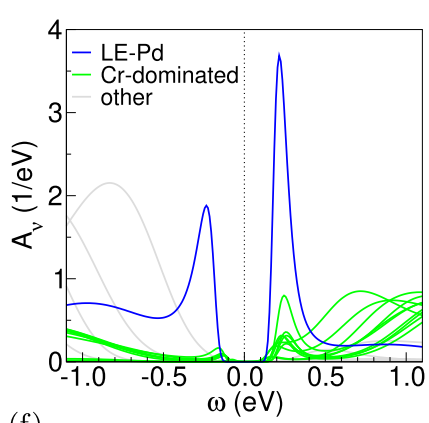

(f)

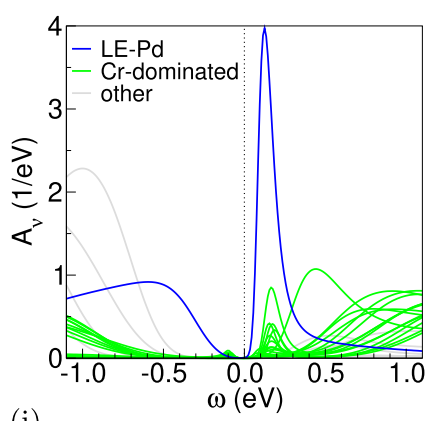

(i)

FIG. 5. Spectral data of the designed heterostructures: (a)-(c) $\mathrm{Pd}_{2} \mathrm{AgCrO}_{2}$, (d)-(f) $\mathrm{PdAgCrO}_{2}$, and (g)-(i) $\mathrm{PdAg}_{2} \mathrm{CrO}_{2}$ at $T=290 \mathrm{~K}$. (a), (d), (g) Spectral function $A(\mathbf{k}, \omega)$ along high-symmetry lines in the $k_{z}=0$ plane of reciprocal space (left) and $\mathbf{k}$-integrated site- and orbital-projected spectral function (right). (b), (e), (h) Fermi surface for $k_{z}=0$ within the first Brillouin zone (green hexagon). (c), (f), (i) k-integrated Bloch contribution $A_{v}(\omega)$ with characterization of dominance. 


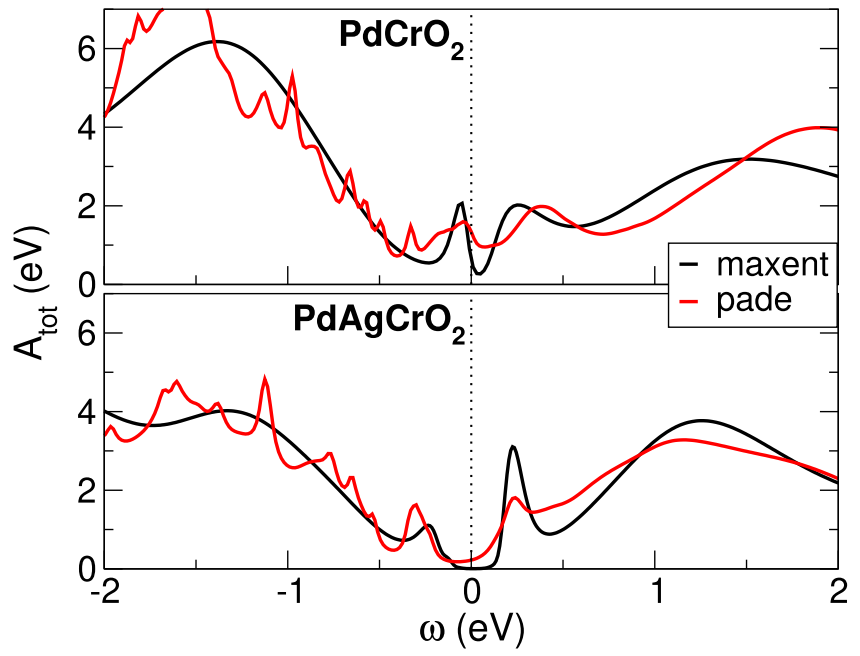

FIG. 6. Comparison between the total $\mathbf{k}$-integrated spectral functions obtained from analytical continuation via maximum entropy and via Padé scheme for $\mathrm{PdCrO}_{2}$ (top) and $\mathrm{PdAgCrO}_{2}$ (bottom). The displayed Padé data results from a straightforward $\mathbf{k}$ sum of $A(\mathbf{k}, \omega)$ on a uniform $\mathbf{k}$ mesh in the respective Brillouin zone.

negative $E_{\text {form }}$; thus the considered phases are stable against decomposition into a mixture of the bulk phases. Within DFT+DMFT, only the P2A structure has a negative formation energy, but the absolute values of the order of $\sim 10 \mathrm{meV} /$ atom are rather small throughout the series. Note that the given $E_{\text {form }}^{(n m)}$ should only be interpreted as a rough guide for phase stability, since the latter depends furthermore on the temperature window, the entropy, the vibrational free energy, and further nanostructuring effects, i.e., given through thin-film architectures. Therefore, in summary, we consider the given $\mathrm{Pd}_{n} \mathrm{Ag}_{m} \mathrm{CrO}_{2}$ phases as indeed serious candidates for stable heterostructures in experiment.

In bulk $\mathrm{PdCrO}_{2}$, the $\mathrm{CrO}_{2}$ layers apparently do not severely harm the itinerancy of the free Pd electron; the established Mott-insulating layers even tend to stabilize the weakly correlated cPd dispersion (see the last paragraph of Sec. III A). The present heterostructuring then formally poses a rather unique canonical problem: the single highly itinerant electron, derived from the Pd layer, faces additional strong driving towards nonitinerant behavior imposed outside its hosting layer via the blocking layers of coupled $\mathrm{Ag}-\mathrm{CrO}_{2}$ kind. Standard mechanisms of creating "heaviness" for such an electron are not easily applicable, yet simply ignoring those new boundary conditions isn't an option either. How does the electron cope with the new situation at room temperature?

The spectral information for the three heterostructures is summarized in Fig. 5. From the k-resolved low-energy dispersions in Figs. 5(a), 5(d) and 5(g), nothing spectacular seems to happen; there are still similar "low-energy bands" at $\varepsilon_{\mathrm{F}}$, reminiscent of the $\mathrm{PdCrO}_{2}$ bulk case. However surprisingly, the k-integrated spectra [see also Figs. 5(c), 5(f) and 5(i)] of all three designed heterostructures show a pseudogap-like structure reaching zero spectral weight at $\varepsilon_{\mathrm{F}}$. This gap feature is of size $\sim 0.2 \mathrm{eV}$ for P2A and PA, and about $\sim 0.1 \mathrm{eV}$ for PA2. It is important to point out that these at first seemingly contradicting findings of $\mathbf{k}$-resolved vs $\mathbf{k}$-integrated spectra

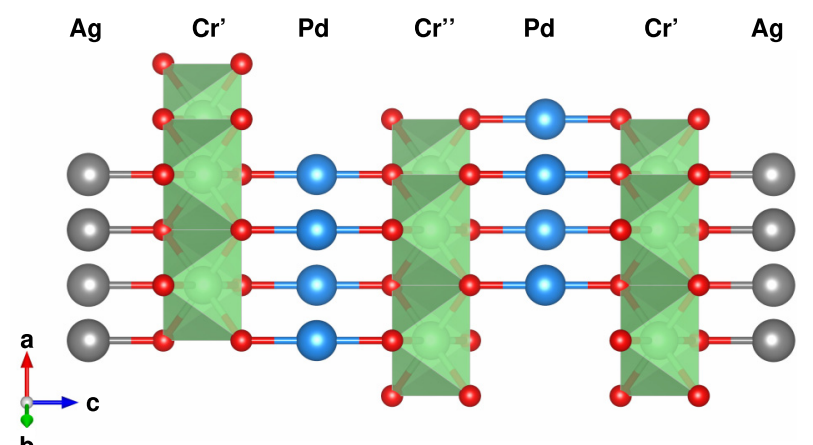

(a)

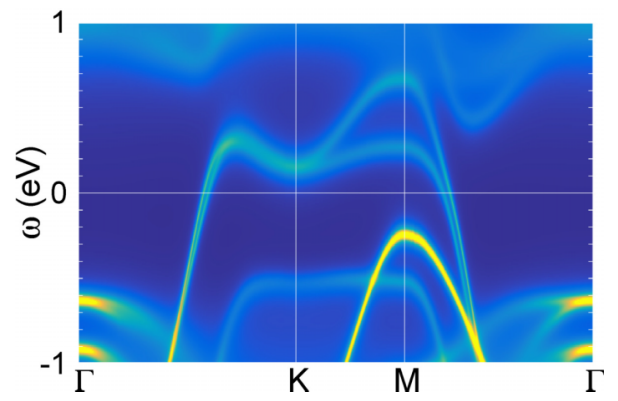

(b)

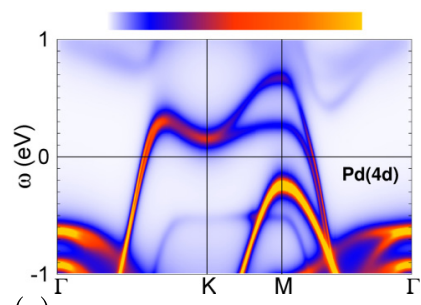

(c)

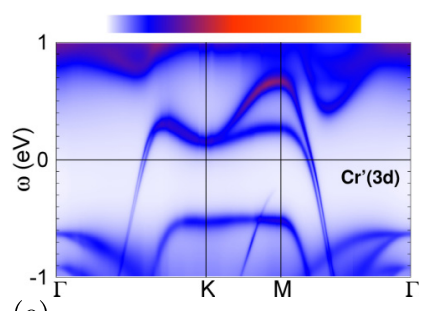

(e)

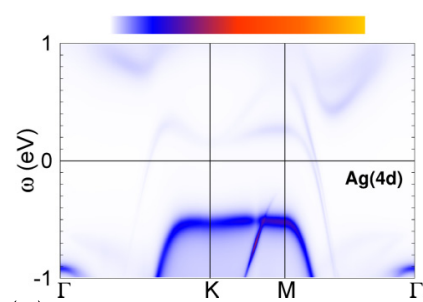

(d)

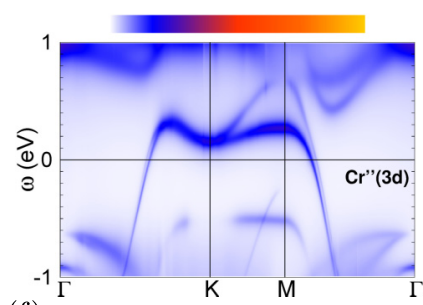

(f)

FIG. 7. Details on the $\mathrm{Pd}_{2} \mathrm{AgCrO}_{2}$ physics. (a) Crystal structure with vertical $c$ axis, displaying the symmetry-inequivalent $\mathrm{Cr}^{\prime}$ and $\mathrm{Cr}^{\prime \prime}$ layers (color coding as Fig. 1). (b) Low-energy blowup of the k-resolved spectral function along high-symmetry lines. (c)(f) Site- and orbital-resolved spectral-function weight: (c) $\operatorname{Pd}(4 d)$, (d) $\mathrm{Ag}(4 d)$, (e) $\mathrm{Cr}^{\prime}(3 d)$, and (f) $\mathrm{Cr}^{\prime \prime}(3 d)$.

are not an artifact of the analytical continuation from Matsubara to real frequencies, but well reproducible both with maximum entropy as well as with Padé schemes. This is shown in Fig. 6, depicting a comparison of the maximum-entropy vs Padé-derived spectrum for bulk $\mathrm{PdCrO}_{2}$ and heterostructure $\mathrm{PdAgCrO}$. While the straightforward k-integrated Padébased spectrum is more "wiggly," the key result at low energy is identical: whereas in $\mathrm{PdCrO}_{2}$ a quasiparticle feature crosses $\varepsilon_{\mathrm{F}}, \mathrm{PdAgCrO}{ }_{2}$ shows a gap(-like) feature at $\omega=0$.

In fact, there is no contradiction between the $\mathbf{k}$-resolved and the $\mathbf{k}$-integrated spectrum. The physical scenario is best coined by the term correlation-induced semimetal (CIS), 
where electronic correlations suppress the low-energy spectral weight in a nontrivial dispersion setting. In a correlated system, a dispersion always carries spectral weight less than unity (contrary to, e.g., a DFT picture), and in the present case the spectral weight associated with the dispersion crossing $\varepsilon_{\mathrm{F}}$ is exceptionally small at room temperature. Figures 5(c), 5(f) and 5(i) show that most of the expected low-energy spectral weight piles up in sidebands at $\sim \pm 0.2 \mathrm{eV}$. Note that this specific low-energy behavior is due to the unique coupling of originally weakly correlated Pd-based and strongly correlated Cr-based contributions in the $(\mathrm{Pd}, \mathrm{Ag}) \mathrm{CrO}_{2}$ system. This coupling leads to low-energy dispersions of very weak spectral weight, resulting in negligible Fermi-level contribution upon $\mathbf{k}$ integration.

Each Fermi-surface sheet, two in the case of P2A, still effectively encompasses one electron, respectively [cf. Figs. 5(b), 5(e) and 5(h)]. Only for the PA2 heterostructure does the $\Gamma$-centered hole sheet, intriguingly, appear slightly enlarged, and we will come back to that observation in the next section.

A further aspect is noteworthy, and can be elaborated best from inspection of the P2A electronic structure with a subtle twofold dispersion crossing $\varepsilon_{\mathrm{F}}$ [cf. Fig. 7(b)]. Figure 7(a) highlights the fact that there are two symmetry-inequivalent Cr layers: $\mathrm{Cr}^{\prime} \mathrm{O}_{2}$ sandwiched by $\mathrm{Ag}$ and $\mathrm{Pd}$ layers as well as $\mathrm{Cr}^{\prime \prime} \mathrm{O}_{2}$ sandwiched by two Pd layers. The twofold dispersion crossing the Fermi level along $\Gamma-K$ and $M-\Gamma$ is nonsurprisingly of dominant $\operatorname{Pd}(4 d)$ character as shown in Fig. 7(c). The low-energy $\operatorname{Ag}(4 d)$ weight accumulates in an occupied flat-band part along $K-M$ with no relevant contribution right at $\varepsilon_{\mathrm{F}}$ [see Fig. 7(d)]. While the $\mathrm{Cr}^{\prime}$ weight is balanced between the twofold dispersion crossing $\varepsilon_{\mathrm{F}}$, the $\mathrm{Cr}^{\prime \prime}$ weight breaks this symmetry and attains somewhat larger weight on the FS sheet with slightly larger $k_{\mathrm{F}}$ [see Figs. 7(e) and 7(f)]. Hence, within the challenging correlation physics of the present delafossite heterostructures, there is furthermore an intricate hybridization component.

\section{Below room temperature}

The CIS state at room temperature is expectedly the result of a very low coherence scale of an underlying effectively doped Mott-insulating state of the $\mathrm{CrO}_{2}$ layers. The DFT+DMFT calculations at lower temperature are numerically especially demanding for the multisite five-orbital correlated subspaces in the heterostructure design. But indeed, below room temperature, a low-energy resonance emerges in the total spectral function of the delafossite heterostructures [see Fig. 8(a)]. Notably, this resonance is now of dominating $\mathrm{Cr}$ character, and carries sizable Pd weight only for the P2A structure (see Fig. 8, right). Thus the given delafossite heterostructures are rather sensitive to temperature and display a semimetal-to-doped-Mott-insulator transition around $190 \mathrm{~K}$.

A minimal picture behind these numerical results may be as follows. Heterostructuring of $\mathrm{PdCrO}_{2}$ and $\mathrm{AgCrO}_{2}$ leads to an effective doping of the $\mathrm{CrO}_{2}$ layers; thus charge fluctuations between the Pd layers and the latter increase. Since the hopping within the Pd layers does not become heavily dressed in the Hubbard sense, the cPd dispersion is not strongly renormalized. In other words, there is no strong cPd band-

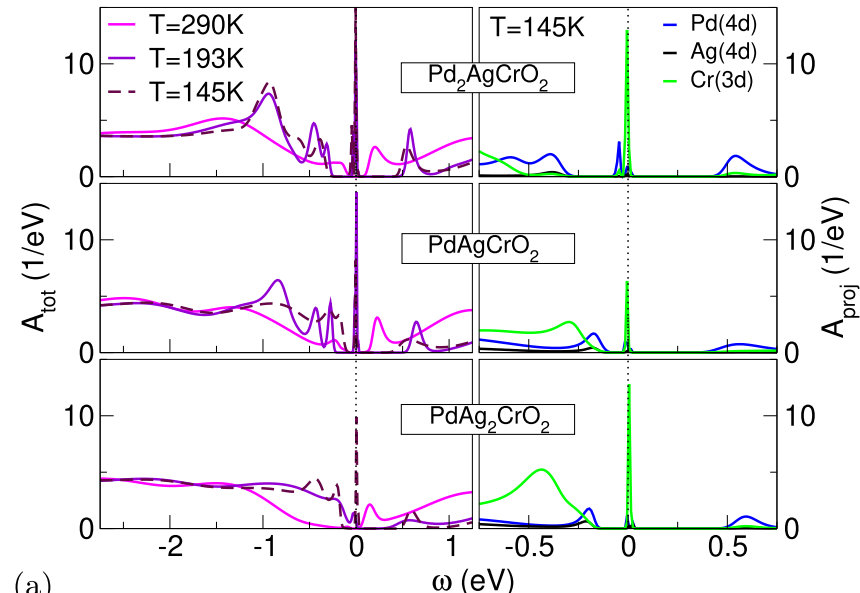

(a)

$\omega(\mathrm{eV})$

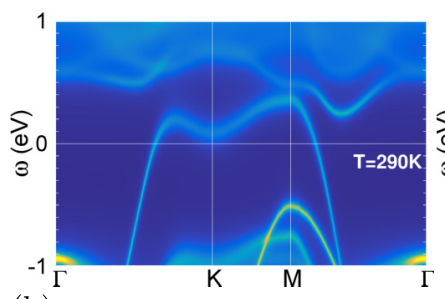

(b)

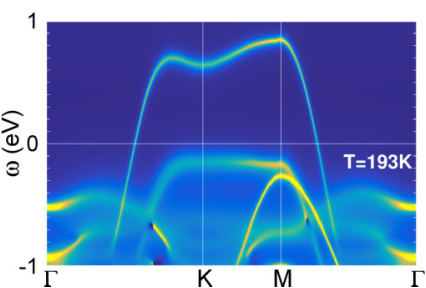

(c)
FIG. 8. Temperature-driven electronic transition in the delafossite heterostructures. (a) Evolution of the total spectral function with temperature throughout the series. Left: $A_{\text {tot }}(\omega)$ for P2A (top), PA (middle), and PA2 (bottom) at $T=290 \mathrm{~K}, 193 \mathrm{~K}$, and $145 \mathrm{~K}$. Right: element-specific projected spectral function at low energy for $T=145 \mathrm{~K}$ (same structure ordering as left part). (b), (c) Low-energy k-resolved spectral function of $\mathrm{PdAg}_{2} \mathrm{CrO}_{2}$ for (b) $T=290 \mathrm{~K}$ and (c) $T=193 \mathrm{~K}$.

narrowing effect, as seen in Figs. 5(a), 5(d) and 5(g). Instead, spectral-weight transfer away from low energy and loss of coherence occurs for an only weakly modified cPd dispersion. Still, the scattering with the doped $\mathrm{CrO}_{2}$ layers is effective in marking a semimetallic scenario. At lower temperatures, the excitations in the doped-Mott layers eventually become coherent and a resonance appears at low energy.

Finally, let us remark on the fermiology of PA2. As obvious from Fig. 8, the PA2 structure develops a low-energy resonance at $T$ lower than the other two heterostructures. At room temperature, the PA2 structure is furthest away from the doped-Mott coherence state. Low-energy coherence of the Mott layers, in the sense previously discussed for Fig. 4, is thus also not yet fully established at that higher $T$. Therefore, the PA2 Fermi surface in Fig. 5(h) shows the deviation from the expected sheet size. Only at lower temperature, when coherence is reinforced, is the expected sheet size for one electron established. This is visualized in the temperature comparison of the low-energy dispersions in Figs. 8(b) and $8(\mathrm{c})$.

\section{CONCLUSIONS AND DISCUSSION}

Delafossite materials, and especially the metallic ones, are not only highly interesting in stoichiometric bulk form. In this paper we have shown that tailored heterostructuring 
of metallic $\mathrm{PdCrO}_{2}$ and insulating $\mathrm{AgCrO}_{2}$ gives rise to a plethora of challenging physics. The explicit aspect of electronic correlation, which is present but to a large extent hidden within the bulk compounds, takes over in the heterostructure phases $\mathrm{Pd}_{n} \mathrm{Ag}_{m} \mathrm{CrO}_{2}$ and renders the demanding rivalry between metallicity and insulating tendencies strikingly obvious. Correlation-induced semimetallic states at room temperature and a transition to a doped Mott-insulating regime with low coherence scale at lower temperature have been revealed. Furthermore, layer-selective hybridization may play a decisive role in order to select the stable many-body states at low energy. Eventual competition with antiferromagnetic spin-ordering tendencies upon further lowering $T$ will even enlarge the scenario of competing many-body instabilities. But already without even considering possible magnetic ordering, there are many relevant degrees of freedom enabling a fascinating playground for very rich physics in designed delafossite heterostructures. An interesting route for investigations could be given by uniaxial-pressure experiments [28-30], either to modify the Hubbard $U$ on $\mathrm{Cr}$ to reach the intricate non-Fermi-liquid regime at smaller $U$ (cf. Fig. 4) or to effectively metallize the Mott layers by effective bandwidth enlargement. Moreover, the construction of explicit topological states within a Mott-critical background may be possible from further tailored heterostructure design.
Still, the question concerning experimental realization of these and further delafossite heterostructures arises. The representation of delafossites with standard growth techniques has been proven challenging. However, the recent growth of $\mathrm{PdCoO}_{2}$ by molecular beam epitaxy [31] is encouraging and should pave the road for a future realization of delafossite heterostructures. In addition, single-layer deposition onto suitable terminated bulk delafossites may already prove helpful in creating exciting electron states on a surface (see, e.g., Ref. [32] for work in that direction).

Let us conclude by the final remark that the key metaldelafossite physics of a crucial coupling between weakly itinerant layers and strongly correlated (Mott) layers connects also to the recent finding of superconductivity in infinite-layer nickelate upon hole doping [33], where similar scenarios might be at play at stoichiometry.

\section{ACKNOWLEDGMENTS}

We thank A. P. Mackenzie and V. Sunko for helpful discussions. Financial support from the DFG LE-2446/4-1 project "Design of strongly correlated materials" is acknowledged. Computations were performed at the JUWELS Cluster of the Jülich Supercomputing Centre (JSC) under Project No. hhh08.
[1] R. D. Shannon, D. B. Rogers, and C. T. Prewitt, Inorg. Chem. 10, 713 (1971); C. T. Prewitt, R. D. Shannon, and D. B. Rogers, ibid. 10, 719 (1971); D. B. Rogers, R. D. Shannon, and C. T. Prewitt, ibid. 10, 723 (1971).

[2] H. Kawazoe, M. Yasukawa, H. Hyodo, M. Kurita, H. Yanagi, and H. Hosono, Nature (London) 389, 939 (1997).

[3] S. Seki, Y. Onose, and Y. Tokura, Phys. Rev. Lett. 101, 067204 (2008).

[4] N. Terada, D. D. Khalyavin, P. Manuel, Y. Tsujimoto, K. Knight, P. G. Radaelli, H. S. Suzuki, and H. Kitazawa, Phys. Rev. Lett. 109, 097203 (2012).

[5] S. Ouyang, Z. Li, Z. Ouyang, T. Yu, J. Ye, and Z. Zou, J. Phys. Chem. C 112, 3134 (2008).

[6] A. P. Mackenzie, Rep. Prog. Phys. 80, 032501 (2017).

[7] R. Daou, R. Frésard, V. Eyert, S. Hébert, and A. Maignan, Sci. Technol. Adv. Mater. 18, 919 (2017).

[8] H. Takatsu, H. Yoshizawa, S. Yonezawa, and Y. Maeno, Phys. Rev. B 79, 104424 (2009).

[9] H.-J. Noh, J. Jeong, B. Chang, D. Jeong, H. S. Moon, E.-J. Cho, J. M. Ok, J. S. Kim, K. Kim, B. I. Min et al., Sci. Rep. 4, 3680 (2014).

[10] F. Lechermann, Phys. Rev. Materials 2, 085004 (2018).

[11] V. Sunko, F. Mazzola, S. Kitamura, S. Khim, P. Kushwaha, O. J. Clark, M. Watson, I. Markovic, D. Biswas, L. Pourovskii et al., Sci. Adv. 6, eaaz0611 (2020).

[12] H. Takatsu, S. Yonezawa, C. Michioka, K. Yoshimura, and Y. Maeno, J. Phys. Conf. Ser. 200, 012198 (2010).

[13] S. Y. Savrasov, G. Kotliar, and E. Abrahams, Nature (London) 410, 793 (2001).

[14] L. V. Pourovskii, B. Amadon, S. Biermann, and A. Georges, Phys. Rev. B 76, 235101 (2007).
[15] D. Grieger, C. Piefke, O. E. Peil, and F. Lechermann, Phys. Rev. B 86, 155121 (2012).

[16] S. G. Louie, K. M. Ho, and M. L. Cohen, Phys. Rev. B 19, 1774 (1979).

[17] B. Meyer, C. Elsässer, F. Lechermann, and M. Fähnle, FORTRAN 90 program for mixed-basis-pseudopotential calculations for crystals, Max-Planck-Institut für Metallforschung, Stuttgart, 1998.

[18] J. P. Perdew, K. Burke, and M. Ernzerhof, Phys. Rev. Lett. 77, 3865 (1996).

[19] B. Amadon, F. Lechermann, A. Georges, F. Jollet, T. O. Wehling, and A. I. Lichtenstein, Phys. Rev. B 77, 205112 (2008).

[20] V. I. Anisimov, D. E. Kondakov, A. V. Kozhevnikov, I. A. Nekrasov, Z. V. Pchelkina, J. W. Allen, S.-K. Mo, H.-D. Kim, P. Metcalf, S. Suga et al., Phys. Rev. B 71, 125119 (2005).

[21] A. N. Rubtsov, V. V. Savkin, and A. I. Lichtenstein, Phys. Rev. B 72, 035122 (2005).

[22] P. Werner, A. Comanac, L. de' Medici, M. Troyer, and A. J. Millis, Phys. Rev. Lett. 97, 076405 (2006).

[23] O. Parcollet, M. Ferrero, T. Ayral, H. Hafermann, I. Krivenko, L. Messio, and P. Seth, Comput. Phys. Commun. 196, 398 (2015).

[24] P. Seth, I. Krivenko, M. Ferrero, and O. Parcollet, Comput. Phys. Commun. 200, 274 (2016).

[25] V. I. Anisimov, I. V. Solovyev, M. A. Korotin, M. T. Czyżyk, and G. A. Sawatzky, Phys. Rev. B 48, 16929 (1993).

[26] J. A. Sobota, K. Kim, H. Takatsu, M. Hashimoto, S.-K. Mo, Z. Hussain, T. Oguchi, T. Shishidou, Y. Maeno, B. I. Min et al., Phys. Rev. B 88, 125109 (2013). 
[27] E. Pavarini, S. Biermann, A. Poteryaev, A. I. Lichtenstein, A. Georges, and O. K. Andersen, Phys. Rev. Lett. 92, 176403 (2004).

[28] S. Riccò, M. Kim, A. Tamai, S. M. Walker, F. Y. Bruno, I. Cucchi, E. Cappelli, C. Besnard, T. K. Kim, P. Dudin et al., Nat. Commun. 9, 4535 (2018).

[29] V. Sunko, E. A. Morales, I. Marković, M. E. Barber, D. Milosavljević, F. Mazzola, D. A. Sokolov, N. Kikugawa, C. Cacho, P. Dudin et al., npj Quantum Mater. 4, 46 (2019).

[30] M. E. Barber, F. Lechermann, S. V. Streltsov, S. L. Skornyakov, S. Ghosh, B. J. Ramshaw, N. Kikugawa, D. A. Sokolov, A. P.
Mackenzie, C. W. Hicks et al., Phys. Rev. B 100, 245139 (2019).

[31] M. Brahlek, G. Rimal, J. M. Ok, D. Mukherjee, A. R. Mazza, Q. Lu, H. N. Lee, T. Z. Ward, R. R. Unocic, G. Eres et al., Phys. Rev. Materials 3, 093401 (2019).

[32] F. Mazzola, V. Sunko, S. Khim, H. Rosner, P. Kushwaha, O. J. Clark, L. Bawden, I. Marković, T. K. Kim, M. Hoesch et al., Proc. Natl. Acad. Sci. USA 115, 12956 (2018).

[33] D. Li, K. Lee, B. Y. Wang, M. Osada, S. Crossley, H. R. Lee, Y. Cui, Y. Hikita, and H. Y. Wang, Nature (London) 572, 624 (2019). 